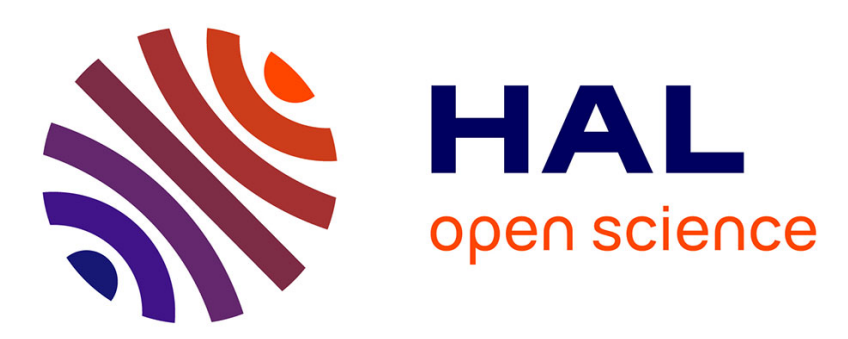

\title{
Anisotropie d'hétérodiffusion moléculaire du $\beta$-naphtol dans le naphtalène monocristallin
}

\author{
A. Dautant, L. Bonpunt, B. Fakhari, N.B. Chanh, Y. Haget
}

\section{To cite this version:}

A. Dautant, L. Bonpunt, B. Fakhari, N.B. Chanh, Y. Haget. Anisotropie d'hétérodiffusion moléculaire $\mathrm{du} \beta$-naphtol dans le naphtalène monocristallin. Journal de Physique Lettres, 1985, 46 (3), pp.117-121. 10.1051/jphyslet:01985004603011700 . jpa-00232486

\section{HAL Id: jpa-00232486 https://hal.science/jpa-00232486}

Submitted on 1 Jan 1985

HAL is a multi-disciplinary open access archive for the deposit and dissemination of scientific research documents, whether they are published or not. The documents may come from teaching and research institutions in France or abroad, or from public or private research centers.
L'archive ouverte pluridisciplinaire HAL, est destinée au dépôt et à la diffusion de documents scientifiques de niveau recherche, publiés ou non, émanant des établissements d'enseignement et de recherche français ou étrangers, des laboratoires publics ou privés. 
Classification

Physics Abstracts

$66.30-61.70$

\title{
Anisotropie d'hétérodiffusion moléculaire du $\beta$-naphtol dans le naphtalène monocristallin
}

\author{
A. Dautant, L. Bonpunt, B. Fakhari, N. B. Chanh et Y Haget \\ Laboratoire de Cristallographie et de Physique Cristalline (*), Université de Bordeaux I, \\ 351, Cours de la Libération, 33405 Talence Cedex, France
}

(Reçu le 31 janvier 1984, révisé le 26 novembre, accepté le 11 décembre 1984)

Résumé. - La diffusion réticulaire de $8{ }^{14} \mathrm{C} \beta$-naphtol dans le naphtalène monocristallin a été mesurée par la méthode de sectionnement comptage dans 2 directions cristallographiques différentes. Les résultats font la preuve de l'existence d'une anisotropie de diffusion moléculaire à $343 \mathrm{~K}$ :

$$
\begin{aligned}
& D_{a}=(12,3 \pm 0,4) \cdot 10^{-17} \mathrm{~m}^{2} \mathrm{~s}^{-1} \\
& D_{c^{\prime}}=(4,3 \pm 0,5) \cdot 10^{-17} \mathrm{~m}^{2} \mathrm{~s}^{-1}
\end{aligned}
$$

Abstract. - By means of a microtome sectioning technique, lattice diffusion at $343 \mathrm{~K}$ of $\beta$-naphthol$8{ }^{14} \mathrm{C}$ into naphthalene single crystals has been measured in two directions. The results reveal the existence of a molecular diffusion anisotropy :

$$
\begin{aligned}
& D_{a}=(12.3 \pm 0.4) \cdot 10^{-17} \mathrm{~m}^{2} \mathrm{~s}^{-1} \\
& D_{c^{\prime}}=(4.3 \pm 0.5) \cdot 10^{-17} \mathrm{~m}^{2} \mathrm{~s}^{-1}
\end{aligned}
$$

\section{Introduction.}

L'étude de la diffusion dans les monocristaux est un moyen puissant pour caractériser les défauts ponctuels ou étendus qui y sont contenus. Alors que la diffusion dans les cristaux ioniques ou métalliques a été largement exploitée, les recherches sur la diffusion moléculaire dans les cristaux organiques restent rares et souvent orientées vers des matériaux de haute symétrie [1]. Nous avons choisi de prendre pour support de notre étude le naphtalène lequel peut être considéré comme matériau modèle pour la physique moléculaire [2]. De basse symétrie (monoclinique), il est potentiellement apte à présenter une anisotropie de diffusion. Cette anisotropie peut-elle être atteinte expérimentalement ? C'est la question à laquelle nous prétendons répondre ici.

A notre connaissance, les seules études d'anisotropie sur matériaux organiques publiées à ce jour concernent d'une part l'autodiffusion dans l'acide benzoïque (monoclinique) et d'autre part l'auto et l'hétérodiffusion à dilution infinie dans l'anthracène (également monoclinique). Dans le premier cas, Mc Ghie et Sherwood [3] ont conclu que les coefficients de diffusion selon les

(*) Laboratoire Associé au C.N.R.S., LA 144. 
directions [010] et [001] sont quasi identiques. Dans le second cas Reucroft, Kervorkian et Labes [4] montrent que si l'anisotropie d'autodiffusion est très faible, celle d'hétérodiffusion du phénanthrène dans l'anthracène est plus marquée : à $0,97 T_{\mathrm{F}}$ la diffusion est environ deux fois plus rapide perpendiculairement au plan (0k0) qu'au plan $(00 l)$.

Nous avons choisi d'essayer de mettre en évidence une éventuelle anisotropie de diffusion moléculaire dans le naphtalène monocristallin par le biais d'une étude d'hétérodiffusion à dilution infinie du $\beta$-naphtol marqué au carbone 14.

\section{Procédure expérimentale.}

Déposer une couche infiniment mince de $\beta$-naphtol contenant des molécules marquées sur la face cristallographique choisie d'un monocristal de naphtalène soigneusement caractérisé, effectuer un recuit de diffusion à température contrôlée $T$, déterminer le profil de pénétration du traceur à l'intérieur du monocristal au bout du temps $t$, exploiter mathématiquement ce profil pour en tirer les coefficients caractéristiques, tel est le cheminement d'une expérimentation d'hétérodiffusion à dilution infinie.

\subsection{CARACTÉRISATION DES MATÉRIAUX.}

- Le $\beta$-naphtol- ${ }^{14} \mathrm{C}$ nous est livré en solution méthanolique par le C.E.A. (activité spécifique $\left.14 \mathrm{mCi} . \mathrm{mole}^{-1}\right)$.

- Les monocristaux de naphtalène nous sont fournis par le professeur Karl. Ils sont préparés par méthode de Bridgman à partir de produit préalablement purifié par sublimation et fusion de zone [5]. Une première sélection est effectuée sur des critères de pureté chimique ne retenant que ceux dont le taux d'impuretés (impureté principale : le thionaphtène) est inférieur à $600 \mathrm{ppm}\left({ }^{1}\right.$ ) (analyses par spectrométrie de masse et chromatographie en phase vapeur couplées). La qualité cristalline est notre second critère de choix. Les caractérisations sont essentiellement optiques et ont porté sur les échantillons taillés en lames à faces parallèles selon les faces de clivage (001). Ne sont retenus que les cristaux dont le taux d'émergences de dislocations n'excède pas $10^{9}$ à $10^{10}$ par $\mathrm{m}^{2}$ pour les figures d'attaque réalisées à l'acide sulfurique.

2.2 PRÉPARATION DES ÉCHANTILlONS DE DIFFUSION. - Le repérage des axes cristallographiques des cristaux finalement retenus est réalisé par méthodes optiques et cristallographiques essentiellement à partir de clichés de Laüe en transmission avec analyse assistée par ordinateur en utilisant un programme mis au point par Laugier et Filhol [6]. Nous avons utilisé la description cristallographique suivante du naphtalène : $\mathrm{P} 2_{1} / a ; Z=2 ; a=8,262(3) ; b=5,984(3) ; c=$ $8,117(5) \AA ; \beta=116,02(2)^{\circ}$. La taille est ensuite effectuée au moyen d'une scie à fil diamanté de $0,25 \mathrm{~mm}$ de diamètre. Pour terminer la face sélectionnée pour le dépôt est rectifiée à l'aide d'un microtome.

Les cristaux ainsi préparés d'environ $8 \times 8 \times 5 \mathrm{~mm}^{3}$ sont soumis à des recuits de "guérison " environ $150 \mathrm{~h}$ à $352 \mathrm{~K}$ (ce qui correspond à $0,996 T_{\mathrm{F}}$ ) suivi d'un refroidissement très lent. Un tel traitement selon Sherwood et White [7] a pour effet de diminuer notablement le taux de dislocations.

2.3 DÉPÔT DE TRACEUR - TRAITEMENT DE DIFFUSION. - Une fine couche de traceur est déposée par évaporation sous vide sur une lamelle de verre restant en contact avec la surface de cristal pendant toute la durée du traitement de diffusion lequel s'effectue à $T_{\mathrm{D}}=343 \mathrm{~K}\left(0,970 T_{\mathrm{F}}\right)$ avec des temps $t$ variant de 250 à $550 \mathrm{~h}$.

( $\left.{ }^{1}\right)$ Le Professeur Karl prépare couramment des monocristaux de naphtalène dont la pureté est de l'ordre de $1 \mathrm{ppm}$. Ici, la nécessité d'avoir un très gros monocristal dans un temps relativement court nous a contraint à être moins exigeants. 
2.4 ANALYSES. - Après élimination des effets de bords, le cristal est sectionné au microtome en tranches de $4 \mu \mathrm{m}$ d'épaisseur parallèlement au front de diffusion. Chaque tranche, mise en solution éthanolique, fait alors l'objet d'une double analyse : la première consiste à mesurer sa radioactivité grâce à un compteur à scintillation liquide, la seconde est une analyse au spectromètre UV qui, en fournissant la teneur globale en molécules naphtaléniques de la solution permet de normaliser l'activité mesurée de chaque tranche de la découpe et donc de s'affranchir des inévitables pertes de produit lors des sectionnements. On détermine ainsi le profil de diffusion $c \equiv k A=f(x)$ où $c$ est la concentration, en molécules marquées à la pénétration $x$ et $A$ l'activité normalisée de la tranche correspondante. Si la diffusion réticulaire est seule en jeu ou encore si elle est fortement majoritaire alors :

$$
c=\left[M /(\pi D t)^{1 / 2}\right] \exp \left[-x^{2} /(4 D t)\right]
$$

où $M$ est la quantité de matière radioactive par unité de surface située à $x=0$ au temps $t=0$. L'exploitation du profil expérimental doit donc permettre d'atteindre le coefficient d'hétérodiffusion à dilution infinie $D$ dans la direction de diffusion étudiée. Toutefois nous allons woir qu'il y a lieu de procéder dans notre cas à des corrections préalables sur les données brutes.

\section{Résultats.}

Nous avons étudié la diffusion dans deux directions orthogonales : l'une perpendiculaire au plan de clivage $(00 l)$ c'est-à-dire selon la direction $c^{\prime}$ l'autre selon l'axe $a$. Le tracé des courbes $\log A=f\left(x^{2}\right)$ a montré, dans tous les cas, une portion initiale linéaire; néanmoins, il apparaît que les graphes présentent toujours une courbure positive pour les valeurs de $x$ plus élevées. Un exemple est illustré à la figure 1. Ceci est le signe d'une participation des défauts étendus au phénomène observé. Les queues de courbe obéissant à une représentation linéaire en $\log A=$ $f(x)$ (voir Fig. 1) nous avons fait nôtres les corrections de Le Claire et Rabinovitch [8,9] pour soustraire la contribution $A_{\mathrm{d}}$ des dislocations à la diffusion observée et donc pour atteindre la diffusion réticulaire seule.

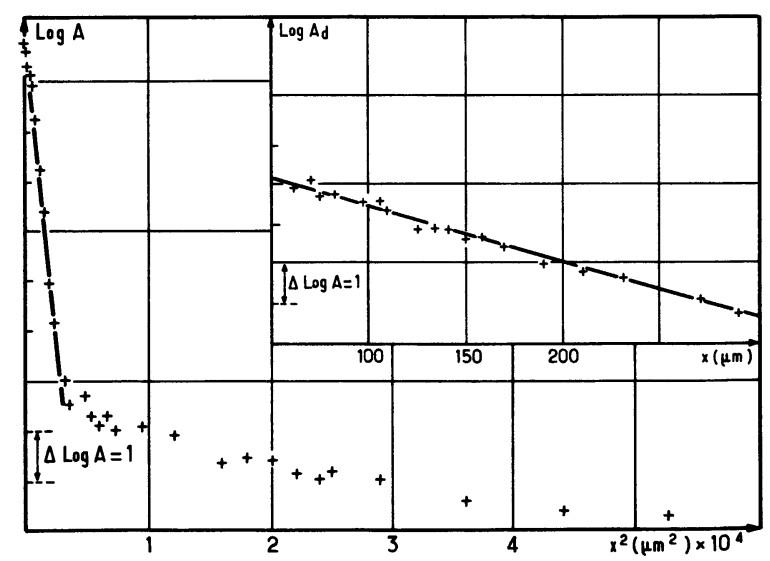

Fig. 1. - Exemple de profil de diffusion du $\beta$-naphtol marqué dans le naphtalène monocristallin (diffusion dans la direction $a$, à $T=343 \mathrm{~K}$ pendant $t=255 \mathrm{~h}$ ); la queue de courbe est également donnée en $\log A=f(x)$.

[An example of diffusion profile of radioactive $\beta$-naphthol in monocrystalline naphthalene (diffusion in the $a$ direction, at $T=343 \mathrm{~K}$ for $t=255 \mathrm{~h}$ ); the tail is also plotted as $\log A$ versus $x$.] 
Une étude préliminaire effectuée au Laboratoire [10] ayant montré que perpendiculairement à $(00 l)$ le coefficient de diffusion est de l'ordre de $10^{-17} \mathrm{~m}^{2} \mathrm{~s}^{-1}$ cela signifiait que nous devions multiplier les expériences pour atteindre des précisions acceptables. Nos résultats, donnés à la figure 2, portent sur 4 expérimentations indépendantes. Dans cette présentation, nous nous sommes affranchis de la notion temps en prenant pour abscisse $x^{2} / t$ où $t$ est le temps de diffusion. Les coordonnées $\log \left(A-A_{\mathrm{d}}\right)$ étant définies à une constante près, nous avons ramené les différents ensembles à une même activité initiale arbitraire. La totalité des points ainsi obtenus, traitée par régression linéaire, conduit à la valeur :

$$
D_{c^{\prime}}=(4,3 \pm 0,5) \cdot 10^{-17} \mathrm{~m}^{2} \mathrm{~s}^{-1}
$$

avec un coefficient de corrélation $r=0,93$ portant sur 26 points.

Nous avons procédé de la même façon pour les résultats concernant la diffusion selon la direction $a$ pour laquelle nous avons limité à 2 le nombre d'expérimentations indépendantes compte

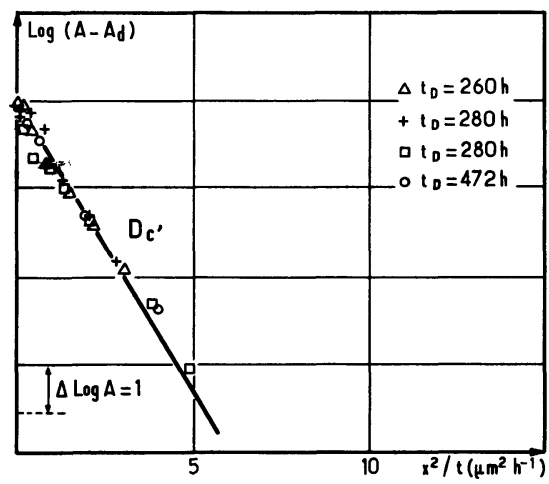

Fig. 2. - Diffusion réticulaire selon la direction $c^{\prime}$.

[Lattice diffusion in the $c^{\prime}$ direction.]

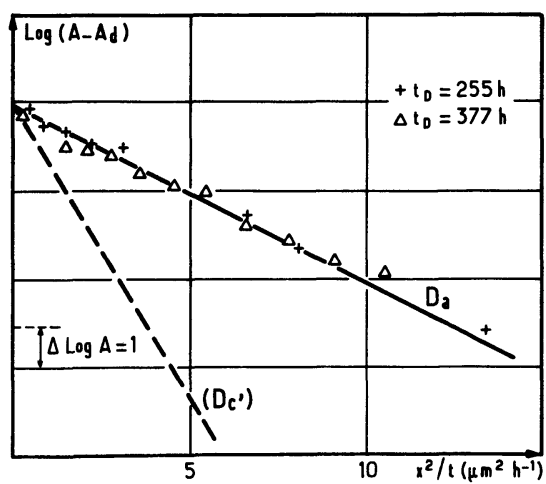

Fig. 3. - Diffusion réticulaire selon la direction $a$ avec le rappel (en pointillés) du résultat relatif à la direction $c^{\prime}$.

[Lattice diffusion in the $a$ direction. For the sake of comparison, the result corresponding to the $c^{\prime}$ direction is given again (dashed line).] 
tenu du fait qu'ici chacune d'elle conduit à une valeur relativement précise du coefficient de diffusion. Par régression linéaire, on aboutit à :

$$
D_{a}=(12,3 \pm 0,4) \cdot 10^{-17} \mathrm{~m}^{2} \mathrm{~s}^{-1}
$$

avec un coefficient $r=0,984$ portant sur 19 points.

\section{Conclusion.}

Au travers de l'analyse de la diffusion de traces de $\beta$-naphtol marqué dans le naphtalène, nos résultats font la preuve de l'existence d'une anisotropie de diffusion réticulaire dans le naphtalène monocristallin puisque la mobilité étudiée est presque 3 fois plus forte dans la direction $a$ que dans la direction $c^{\prime}$. Nous travaillons actuellement à la détermination complète du tenseur de diffusion à $343 \mathrm{~K}$. Nous serons alors aptes à discuter les résultats ci-dessus et à les comparer avec ceux obtenus par ailleurs pour l'autodiffusion du naphtalène.

\section{Remerciements.}

Nous tenons à remercier le Professeur N. Karl du Physikalisches Institut de l'Université de Stuttgart pour les monocristaux de naphtalène qu'il a bien voulu préparer à notre intention et pour les discussions que nous avons pu avoir avec lui.

\section{Bibliographie}

[1] Chezeau, J. M. et Strange, J. M., Phys. Rep. 53 (1979) et les références citées.

[2] Kitaigorodsky, A. I., Molecular crystals and molecules (Academic Press, New York) 1973, p. 357.

[3] McGhie, A. R., Sherwood, J. N., Trans. Faraday Soc. 67 (1971) 3364.

[4] Reucroft, P. J., Kervorkian, H. K., Labes, M. M., J. Chem. Phys. 44 (1966) 4416.

[5] KarL, N., Crystals : Growth, Properties and Applications (Springer-Verlag Berlin, Heidelberg, New York) 1980 , p. 1-100.

[6] Laugier, J., Filhol, A., J. Appl. Cryst. 16 (1983) 281.

[7] Sherwood, J. N., White, D. J., Philos. Mag. 15 (1967) 745.

[8] Le Claire, A. D., Rabinovitch, A., J. Phys. C 14 (1981) 3863.

[9] Le Claire, A. D., Rabinovitch, A., J. Phys. C 15 (1982) 3455.

[10] Meynard, J., Thèse Bordeaux (1981). 